single-vessel CAD 212 (396) and more severe disease 170 (327) ms²; $\mathrm{p}$-value for trend $=0.003$. There was a similar reduction in LF power regardless of the anatomical location of coronary stenoses (see Abstract 61 figure 1). Comparing patients with $\mathrm{LF}<250$ and $\geq 250 \mathrm{~ms}^{2}$, the OR for obstructive CAD was $2.42,95 \%$ CI 1.33 to $4.38(\mathrm{p}=0.004)$ after adjusting for risk factors, medications and heart rate. No interactions were noted in sub-group analysis of age, gender, diabetes, prior cardiovascular disease or high-sensitivity CRP. In addition, HRV added to risk prediction irrespective of baseline Framingham risk $(p<0.0001)$.

Conclusion Low HRV is strongly predictive of angiographic coronary disease regardless of other comorbidities and is clinically useful as a risk predictor in patients with sinus rhythm.

\section{WILL THE NEW EUROPEAN AF GUIDELINES LEAD TO MORE PATIENTS RECEIVING ORAL ANTICOAGULATION THERAPY?}

doi:10.1136/heartjnl-2011-300198.62

T Kaier, R Williams, S Khan. West Middlesex University Hospital, London, UK

Atrial fibrillation (AF) confers a 5-fold risk of stroke and the risk of death from AF-related stroke is doubled; it is the most common sustained cardiac arrhythmia, occurring in $1 \%-2 \%$ of the general population. Meta-analysis of anti-platelet therapy demonstrate a non-significant $19 \%$ reduction in the incidence of stroke, proving oral anticoagulation (OAC; such as warfarin) to be far superior (64\% relative risk reduction) in stroke prevention. Recent guidelines have been published by the European Society of Cardiology (ESC) which focus on the most effective antithrombotic therapy in AF and propose a new risk scoring system, the CHA2DS2-VASc score. In our institution the prescription and documentation of antithrombotic therapy in AF has been the focus of a previous audit that demonstrated poor compliance with the guidelines and documentation of decision making. The focus of this audit was twofold: first to determine whether compliance with the guidelines and documentation had improved and second determine the effect of the new risk scoring system on prescription of OAC. A random $10 \%$ of cases of patients discharged with a coding diagnosis of AF were selected (125 cases). They were risk assessed using the NICE 2006 stroke risk stratification, CHADS2 and CHA2DS2-VASc score. In all cases the agent used for thromboprophylaxis was reviewed as to whether NICE recommendations had been met. The scoring systems were compared to identify patients in whom the ESC guidelines would change treatment-ie, OAC instead of Aspirin or as first line antithrombotic therapy. Of the 125 selected case notes 114 arrived in time for analysis; out of these 8 were excluded due to erroneous coding as AF. 106 patients were risk stratified, of whom $68.22 \%$ (73) were high risk, $28.04 \%$ (30) moderate and $2.80 \%$ (3) low risk according to NICE guidelines. $74.77 \%$ (80) scored 2 or more points on the CHADS2 risk assessment-this number increased to $93.46 \%$ (100) if CHA2DS2-VASc was applied, for whom OAC would be the recommended antithrombotic therapy (see Abstract 62 figure 1). Only $57.50 \%$ or 61 patients were on the appropriate choice of thromboprophylaxis if the NICE guideline was used as risk assessment. $73.77 \%$ (45) of patients had no formal documentation why NICE guideline had not been followed; this mostly comprised patients who were on Aspirin but, correctly risk assessed, were candidates for OAC. We extrapolate our findings to suggest that in an average sized DGH, 200 patients more per year would be considered high thromboembolic risk and hence appropriate for OAC. This audit shows that the rate of appropriate anti-coagulation among patients with $\mathrm{AF}$ is still low and could be improved further. The new ESC guidelines add to this challenge as significantly more patients will be considered for OAC therapy.

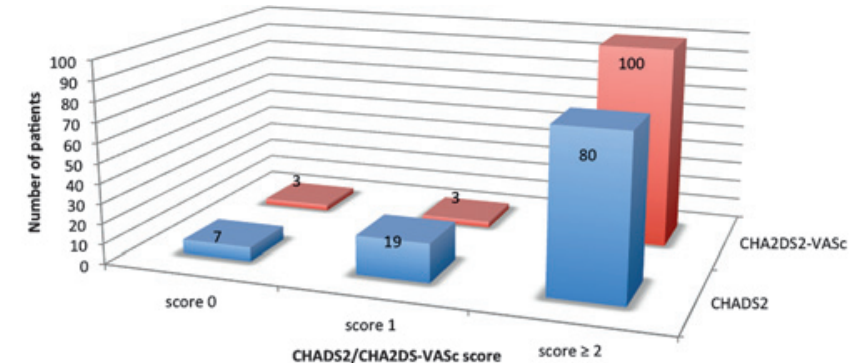

\begin{tabular}{|l|c|c|c|}
\cline { 2 - 4 } \multicolumn{1}{c|}{} & score 0 & score 1 & score $\geq 2$ \\
\hline \hline "CHADS2 & 7 & 19 & 80 \\
\hline \hline "CHA2DS2-VASC & 3 & 3 & 100 \\
\hline
\end{tabular}

Abstract 62 Figure 1 Comparison CHADS2 \& CHA2DS2-VASc scores.

\section{IMPLICATIONS OF A LIKELIHOOD BASED APPROACH TO DIAGNOSTIC TESTING IN CORONARY ARTERY DISEASE: IMPACT OF THE NEW NICE GUIDELINES}

doi:10.1136/heartjnl-2011-300198.63

I U Haq, J S Skinner, P C Adams. Royal Victoria Infirmary, Newcastle upon Tyne, UK

Background The NICE clinical guideline for chest pain of recent onset (NCG 95) published in March 2010 recommends diagnosing angina based on clinical assessment and likelihood of coronary artery disease (CAD). If the estimated likelihood of CAD is $61 \%-$ $90 \%$, coronary angiography should be offered. If $30 \%-60 \%$, functional imaging should be offered, and if 10\%-29\%, CT calcium scoring should be offered. We determined the number and types of different investigations to diagnose coronary artery disease in patients referred with suspected cardiac pain before the publication of NCG 95 and compared this with the predicted investigations after the application of the guidelines.

Methods Data was collected prospectively in a bespoke database for patients referred to the Rapid Access Chest Pain Clinic, Newcastle upon Tyne, UK. Patients with chest pain of suspected cardiac origin were referred from primary care between February 2002 and March 2010. Patients with previous MI, PCI or CABG were excluded. The analysis comprised 5598 men and women with no past history of coronary disease. Likelihood of CAD was calculated by the Pryor equation using the variables age, sex, type of chest pain (typical or atypical), ECG $Q$ waves, smoking, hyperlipidaemia, diabetes and ST/T changes on ECG. The main outcome measures were actual and predicted future frequency of exercise tests, CT coronary angiograms, functional imaging tests and invasive coronary angiograms by pretest likelihood of coronary artery disease.

Results The proportion of the study population before and after the guidelines undergoing exercise testing was $50.1 \%$ vs $0.0 \%$; for calcium score/CT coronary angiography $0.0 \%$ vs $14.7 \%$; for functional imaging $25.6 \%$ vs $13.4 \%$; and for invasive coronary angiography $15.3 \%$ vs $25.8 \%$. The proportion not requiring further testing was unchanged (30.0\% vs $31.0 \%$ ).

Conclusions Application of NICE CG95 will change the investigation of patients with chest pain substantially. A significant reallocation of resources will be required. Exercise testing will be replaced by anatomic or functional imaging. CT coronary angiography will play an important role and replace functional imaging in some patients. Invasive angiography will take on a more important role in the diagnosis of coronary artery disease. It will, however, empower us to reassure almost a third of referrals that they do not have angina on clinical assessment alone. 